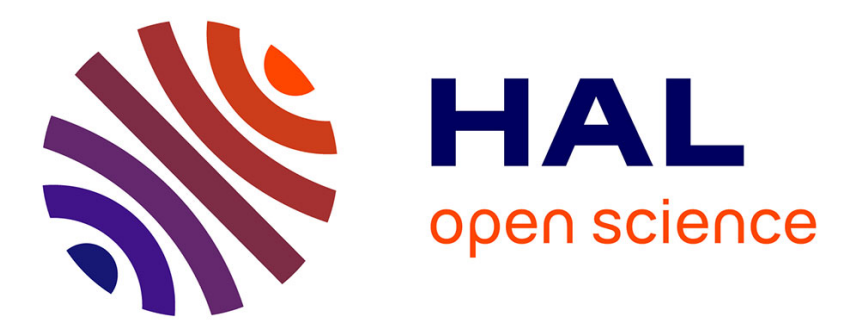

\title{
Ultrafast Bessel beams for high aspect ratio taper free micromachining of glass
}

\author{
M.K. Bhuyan, F. Courvoisier, P.-A. Lacourt, M. Jacquot, L. Furfaro, M. J. \\ Withford, J.M. Dudley
}

\section{To cite this version:}

M.K. Bhuyan, F. Courvoisier, P.-A. Lacourt, M. Jacquot, L. Furfaro, et al.. Ultrafast Bessel beams for high aspect ratio taper free micromachining of glass. Conference on Nonlinear Optics and Applications IV, Apr 2010, Bruxelles, Belgium. pp.77281V, 10.1117/12.854767 . hal-00563255

\section{HAL Id: hal-00563255 \\ https://hal.science/hal-00563255}

Submitted on 12 Apr 2021

HAL is a multi-disciplinary open access archive for the deposit and dissemination of scientific research documents, whether they are published or not. The documents may come from teaching and research institutions in France or abroad, or from public or private research centers.
L'archive ouverte pluridisciplinaire HAL, est destinée au dépôt et à la diffusion de documents scientifiques de niveau recherche, publiés ou non, émanant des établissements d'enseignement et de recherche français ou étrangers, des laboratoires publics ou privés. 


\title{
Ultrafast Bessel beams for high aspect ratio taper free micromachining of glass
}

\author{
M. K. Bhuyan ${ }^{\mathrm{a}}$, F. Courvoisier ${ }^{\mathrm{a} *}$, P.-A. Lacourt ${ }^{\mathrm{a}}$, M. Jacquot ${ }^{\mathrm{a}}$, L. Furfaro ${ }^{\mathrm{a}}$, \\ M. J. Withford ${ }^{\mathrm{b}}$ and J. M. Dudley ${ }^{\mathrm{a}}$ \\ ${ }^{a}$ FEMTO-ST Institute, Department of Optics P.M. Duffieux, UMR CNRS 6174, Université de \\ Franche-Comté, 25030 Besançon, France \\ ${ }^{\mathrm{b}}$ CUDOS and MQ Photonics, Department of Physics, Macquarie University, New South Wales, \\ 2109, Australia
}

\begin{abstract}
Although ultrafast lasers have demonstrated much success in structuring and ablating dielectrics on the micrometer scale and below, high aspect ratio structuring remains a challenge. Specifically, microfluidics or lab-on-chip DNA sequencing systems require high aspect ratio sub-10 $\mu \mathrm{m}$ wide channels with no taper. Micro-dicing also requires machining with vertical walls. Backside water assisted ultrafast laser processing with Gaussian beams allows the production of high aspect ratio microchannels but requires sub-micron sample positioning and precise control of translation velocity.

In this context, we propose a new approach based on Bessel beams that exhibit a focal range exceeding the Rayleigh range by over one order of magnitude. An SLM-based setup allows us to produce a Bessel beam with central core diameter of $1.5 \mu \mathrm{m}$ FWHM extending over a longitudinal range of $150 \mu \mathrm{m}$. A working window in the parameter space has been identified that allows the reliable production of high aspect ratio taper-free microchannels without sample translation. We report a systematic investigation of the damage morphology dependence on focusing geometry and energy per pulse.
\end{abstract}

Keywords: Bessel beams, ultrafast laser, material processing, filamentation

\section{INTRODUCTION}

The field of micro- and nanofluidics exponentially expands its field of applications, from fundamental research purposes to sophisticated micro-Total Analysis Systems ( $\mu$ TAS) with increasingly demanding complexity and finesse for chemical analysis, sensors, drug screening or chemical micro-reactors[1]. More recently, a strong research effort has been dedicated to trapping, detection, manipulation and measurements on single macro-molecules such as DNA, in nanochannels and nano-pores[2][3]. Most complex micro and nano-fluidics components are fabricated in poly(dimethylsiloxane) (PDMS) or poly(methylmetacrylate) (PMMA) since these polymers can be easily processed by conventional lithography or soft lithography techniques with a high throughput [4].

However, these materials suffer from two drawbacks. First, their resistance to solvents is low and many proteins adsorb on these materials. Second, they can hardly be used for engraving photonic components, as femtosecond waveguide burning: many local defects lead to a low optical damage threshold and low transparency. Glass is ideally suited for the integration of optical components and its ultra-low chemical reactivity allows for the realization of combined photonic and fluidic systems [5].

In this context, the machining of long channels in glass with a few micrometers in diameter is a key fabrication step. For this, femtosecond laser processing has attracted much interest since it allows for processing both microchannels and waveguides in the same glass sample [6]. The well defined threshold of femtosecond laser ablation renders the ablation quasi-deterministic and allows for the processing of microstuctures in 3 dimensions. Since its broad range of applications, versatility, fast prototyping ability, femtosecond laser processing is currently an active field of intense research [7]. 
Femtosecond laser microstructuration has been successfully used for microchannels processing in various glasses with high aspect ratio. A two-step technique has been introduced by Marcinkevicius et al [8]: it first consists in a femtosecond laser irradiation of the glass sample. Then, immersion in a fluorhydric acid solution leads to selective etching of the irradiated regions. All kinds of shapes and high aspect ratio were produced with typical channel diameters between 1 and $100 \mu \mathrm{m}$. This approach is very efficient, but chemical etching requires several hours [9] and waveguides can be burned in the same irradiation step as microchannels only with much precautions.

Direct channel drilling in ambient air by femtosecond laser ablation does not allow high aspect ratio ablation. Redeposition, debris evacuation limits the accessible aspect ratio to less than 5 for channels of 10 to $100 \mu \mathrm{m}$ diameter when drilling in ambient air..

Rear-side drilling with water immersion has been introduced by Li et al [10] to answer the problems of producing high aspect ratio microchannels with adaptable diameters, low taper, reduced damaged zone and low number of cracks. Indeed, when the laser processing is performed from the rear surface and followed by translation inward the sample, the translation keeps almost identical ablation parameters along the drilling, that produce channels with low taper. The contact with water at the site of optical breakdown in the transparent sample leads to bubble formation that expels the debris out of the channel, thus avoiding the need for high intensities to extract ablation debris. Microchannels of diameter $5 \mu \mathrm{m}$ with an aspect ratio up to 50 have been demonstrated at an effective translation speed of $0.3 \mu \mathrm{m} / \mathrm{s}$ including waiting times [10]. Ultrasonic wave agitation helps to increase the channel quality and processing speed at the expense of a channel diameter increase [11]. In this configuration, microchannels were processed with diameter on the order of $30 \mu \mathrm{m}$ with an aspect ratio of $\sim 20$ at a speed up to $30 \mu \mathrm{m} / \mathrm{s}$. To the best of the authors' knowledge, the smallest channels diameters with longest channel lengths in glass were obtained by strong focusing, multiple pass, with effective drilling velocities lower than $1 \mu \mathrm{m} / \mathrm{s}$.

Immersion-assisted microdrilling of glass by femtosecond laser has thus been shown as very promising for the prototyping of 3D micro- and submicrometer- channels. However, the effective drilling velocity is rather low and a key experimental condition is to precisely maintain the laser focal spot on the ablation front.

A simple approach to directly process high aspect ratio structures is to use high aspect ratio beams. Bessel beams [12] have been previously used for laser processing in metals [13]. However, this led to channels with taper due to the non-transparency of the material. Although Bessel beams have been used to induce index modification in glass [14], direct drilling with femtosecond Bessel beams in transparent media has never been performed. Besides, the propagation of intense femtosecond laser pulses in the filamentation regime also lead to high aspect ratio beams. Several studies of filamentation applied to micromachining and microstructuring in glass have been realized up to here [15]. UV filaments generated in air have also been demonstrated to generate high aspect ratio holes in $\mathrm{LiNbO}_{3}$ [16]. However, the generation of filaments by Gaussian beams is rather complex to control and difficult to apply to laser micromachining since the plasma channel has not a constant density along the propagation direction due to temporal splitting and nonlinear losses.

More, at high laser power, the beam enters in a multifilamentation regime [17][18].

In this context, we report a novel and experimentally straightforward approach using femtosecond Bessel filaments, that have recently attracted much attention [19-[21] Filaments created by femtosecond Bessel beams have exceptional properties. Indeed, they are stationary solutions to the nonlinear propagation of ultrashort pulses in the presence of nonlinear losses in Kerr media [22]. They do not lead to multifilamentation, even for powers up to 100 critical powers and can provide plasma channels with constant electronic density [20].

We show here that femtosecond Bessel filaments have the potential to answer these problems. We investigate here in which conditions Bessel beams can lead to high aspect ratio drilling in glass with water assistance and investigate the stability conditions of Bessel filaments by means of a systematic study of the damage morphology on energy and beam position in the sample.

\section{EXPERIMENTAL SETUP}

Our experimental setup is shown in Fig. 1a. Here we generated a Bessel beam by using a non-pixelated spatial light modulator to imprint the spatial phase of an axicon onto the beam of a regeneratively amplified $100 \mathrm{fs}$ Ti:Sa laser operating at a central wavelength $800 \mathrm{~nm}$. This Bessel beam was then de-magnified by a factor $1 / \mathrm{M}=110$ using a telescope consisting of a lens of focal distance $\mathrm{f}=1 \mathrm{~m}$ and a $\times 20$ microscope objective (MO). The resulting beam has a Bessel Gaussian profile with a conical angle $\theta=10^{\circ}$, central spot diameter $\mathrm{d}=1.5 \mu \mathrm{m}$ FWHM and depth of focus D $=150 \mu \mathrm{m}$ FWHM . Although the beam has a Bessel-Gauss profile, it will be designated throughout this article by Bessel 
beam. The beam has been characterized by imaging in 3D its intensity distribution by translating a CCD camera associated to a 40x magnification arrangement. The study was conducted with borosilicate glass (Corning 0211) samples of thickness $150 \mu \mathrm{m}$.

\section{FOCUSING GEOMETRY}

The first geometry investigated was the the following: the center of the Bessel beam was placed on the sample center since the FWHM of the Bessel beam is of same size as the sample thickness. Fig $1 \mathrm{~b}$ (top) shows the intensity distribution in the sample.. Fig. 1c (top) shows the corresponding damage in the glass slide realized after an illumination by 500 laser shots. The sample has been imaged from side under a Differential Interference Contrast (DIC) microscope. The fringes on both sides of the glass slide are due to Fresnel interferences from the microscope illumination conditions. The result is clearly unsatisfactory since the damage is not homogeneous along the propagation direction.

Therefore, two other focusing geometries were compared: frontside ablation, corresponding to Fig. 1b (center) and rear side focusing with water immersion (Fig $1 \mathrm{~b}$, bottom). For an easier comparison of the crater profiles, we realized micro-trenches with in-sample plane translation of the Bessel beam. The samples were then cleaved and imaged by Scanning Electron Microscopy (SEM).

Front-side laser machining with Bessel beams has already been used to ablate metals [13]. In this case, the channels produced had a moderate aspect ratio ( 10) and a non-negligible taper which was due to beam truncation by the metallic interface. For glass, we also observed tapering for all front-side trenches realized. A striking example is shown on figure 1c (center). The reason of the tapering is that when the sample is processed in ambient air, the ablation debris are partially redeposited on the sample surface and lower the ablation threshold for following laser pulses. This leads to ablation by the side lobes.

On the contrary, rear side processing under water immersion can produce trenches with vertical walls. A typical example is shown on Fig 1c (bottom) that was obtained with 100 pass and in-plane translation velocity of $0.5 \mathrm{~mm} / \mathrm{s}$. The aspect ratio of the trench is 5 and the walls are parallel within an error bar of $3^{\circ}$. Fig 2 shows the processing of a microchannel in such rear-side focusing conditions without translation. The microchannel length is $83 \mu \mathrm{m}$ and its diameter $2 \mu \mathrm{m}$. 
a) Setup

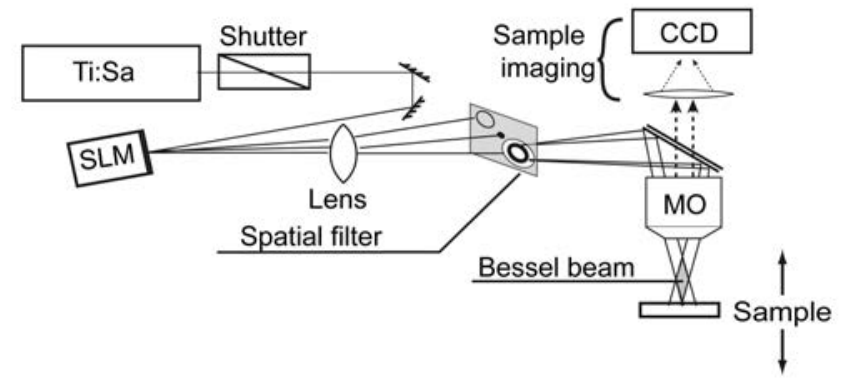

b) Focusing geometry

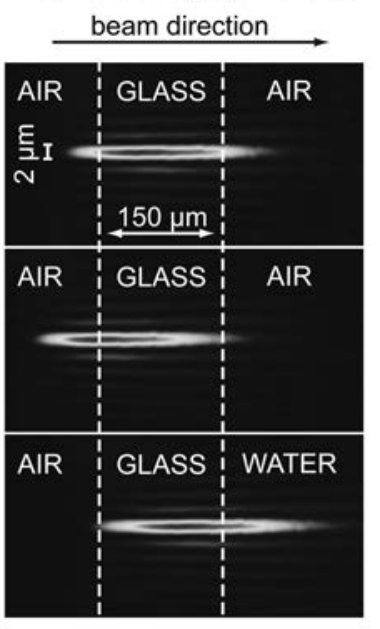

c) Structures

beam direction
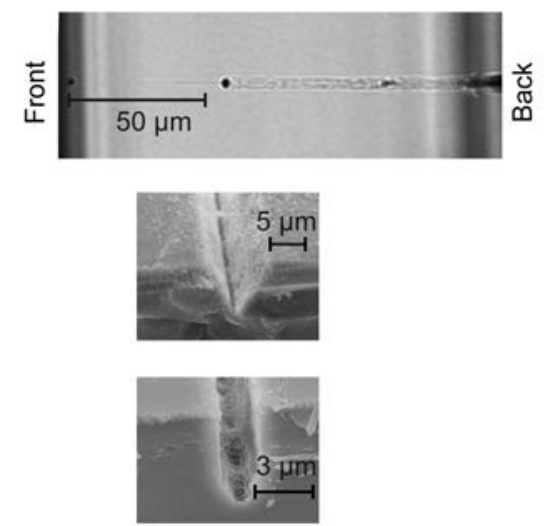

Figure 1 a)experimental setup b-c) comparison of the focusing geometry in the sample and the resulting typical structures produced

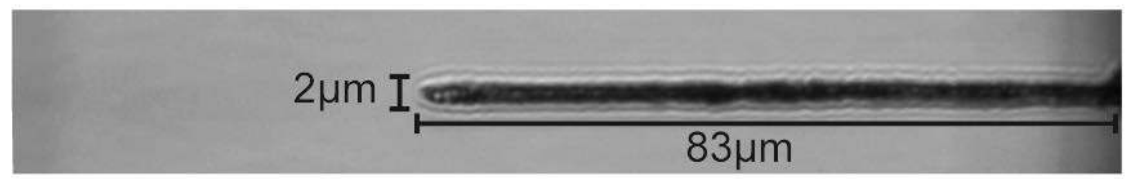

Figure 2: Microchannel processed with 1000 laser shots, without translation, in rear-side focusing conditions. 


\section{STABLE/UNSTABLE DRILLING REGIMES}

Fig 3 shows the evolution of channel diameter and length with energy. It is immediately apparent that for energies between 6.5 and $8 \mu \mathrm{J}$ per pulse, the channels present no taper, as in fig. 2 . Over a threshold energy $8.5 \mu \mathrm{J}$, the mean channel length rapidly reduces and the channel diameter increases. Moreover, in this regime, the error bars largely increase compared to the previous regime and reach more than $80 \%$. In this regime, the channel diameter does not remain constant over the channel length. The difference between these two regimes is attributed to two different regimes of filamentation[23]. Studies of Bessel filaments in water by Polesana et al [20] suggest that in our case, the appearance of an unstable propagation regime is triggered by a contrast of Kerr nonlinearities at the sample entrance surface.

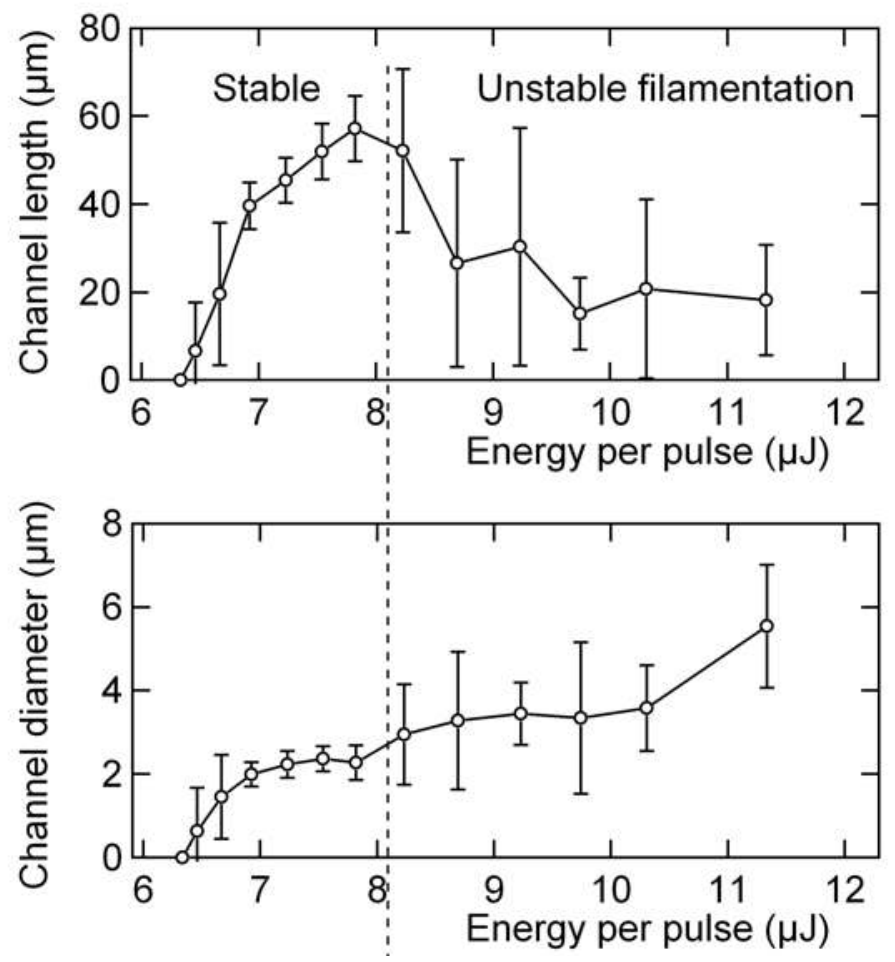

Figure 3 Dependance of channel diameter and length on energy per pulse $(100 \mathrm{~Hz}, 1000$ shots with water immersion, rear side machining without translation) 


\section{DEPENDENCE ON BEAM ONSET}

To get a better insight into the propagation properties, we investigated the damage produced by 1000 shots without water assistance. No deep ablation is produced. Fig 4 shows the evolution of the damage morphology produced by $130 \mathrm{fs}$ laser pulses, for different energies and 4 different beam positions inside the sample.

When the beam onset is at $\mathrm{D}=30 \mu \mathrm{m}$ after the entrance interface, the damage track is continuous, as expected for stable filaments. The result is near identical for $D=0 \mu \mathrm{m}$. When the beam starts before the entrance surface ( $D=-25$ and $\mathrm{D}=-50 \mu \mathrm{m}$ ), the power at the entrance surface is non-negligible. Two different regimes show up. For $\mathrm{D}=-25 \mu \mathrm{m}$, the damage track is not constant: dark spots appear at regularly increasing distance. This behavior is reproducible. For $\mathrm{D}=-$ $50 \mu \mathrm{m}$, the power at the entrance is sufficiently high to produce surface ablation. The ablated site behaves as an obscurant. The beam self-reconstructs afterwards, but regular dark spots are not visible in this regime.

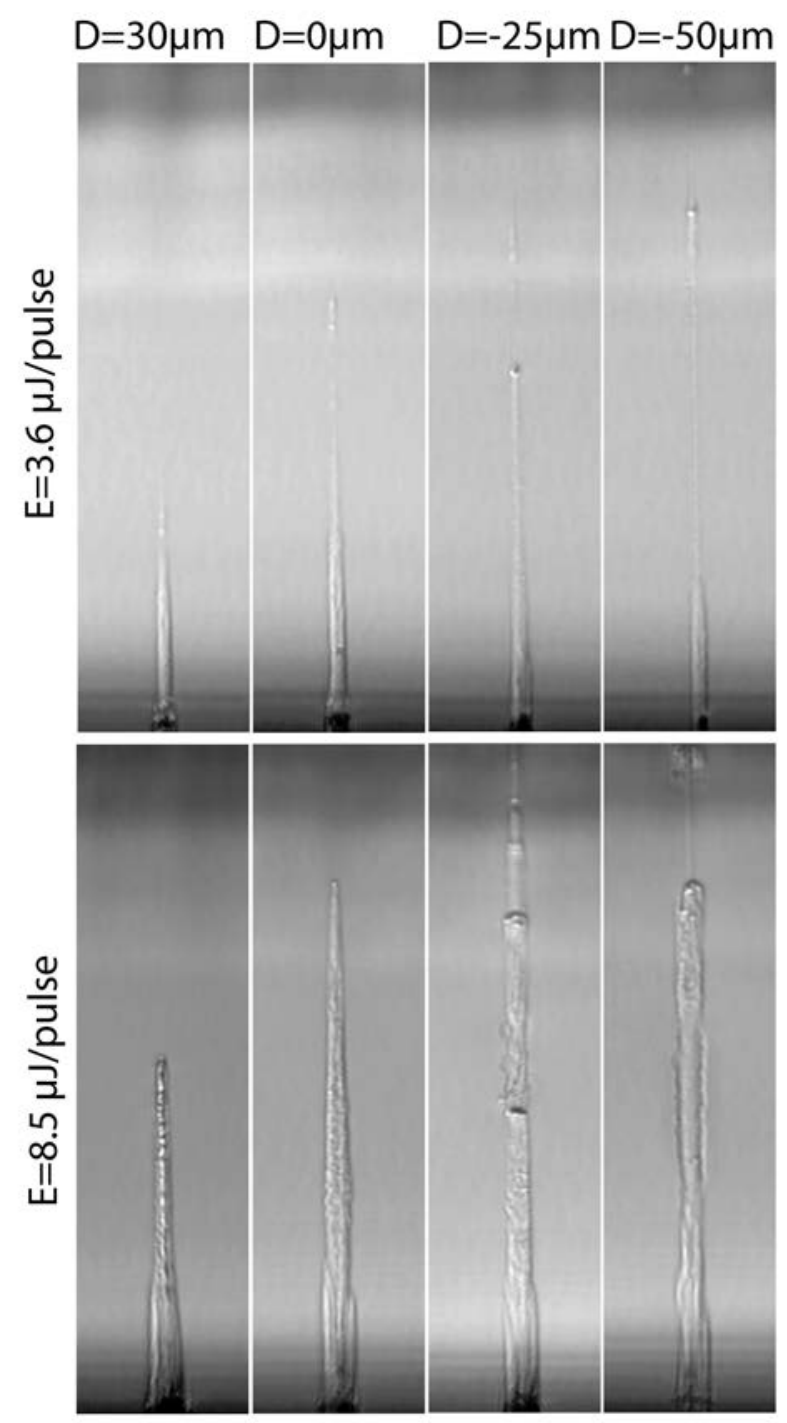

Figure 4 Image with Differential interference contrast microscopy of the damage track produced by 1000 shots, $100 \mathrm{~Hz}, 130 \mathrm{fs}$ laser pulses.

Figure 5 shows the evolution of the damage with increasing pulse energy for fixed beam onset at $\mathrm{D}=-25 \mu \mathrm{m}$, i.e. $25 \mu \mathrm{m}$ before the sample front surface. From 3.2 to $9.1 \mu \mathrm{J} /$ pulse, regular dark spots indicate that plasma density strongly 
oscillates along the propagation. The number of oscillations increases with energy. From $10.3 \mu \mathrm{J} / \mathrm{pulse}$, the morphology of the damage changes: no dark spot is visible. Oscillations are suppressed. Two effects can explain the disappearance of unstability: the energy loss created by surface damage may reduce the nonlinear contrast at the surface, yielding softener input conditions and continuous plasma channel, and/or using higher intensities may help locking the nonlinear dynamical process of single plasma channel generation, as predicted by [20]. Further numerical work is required to better understand these experimental observations.

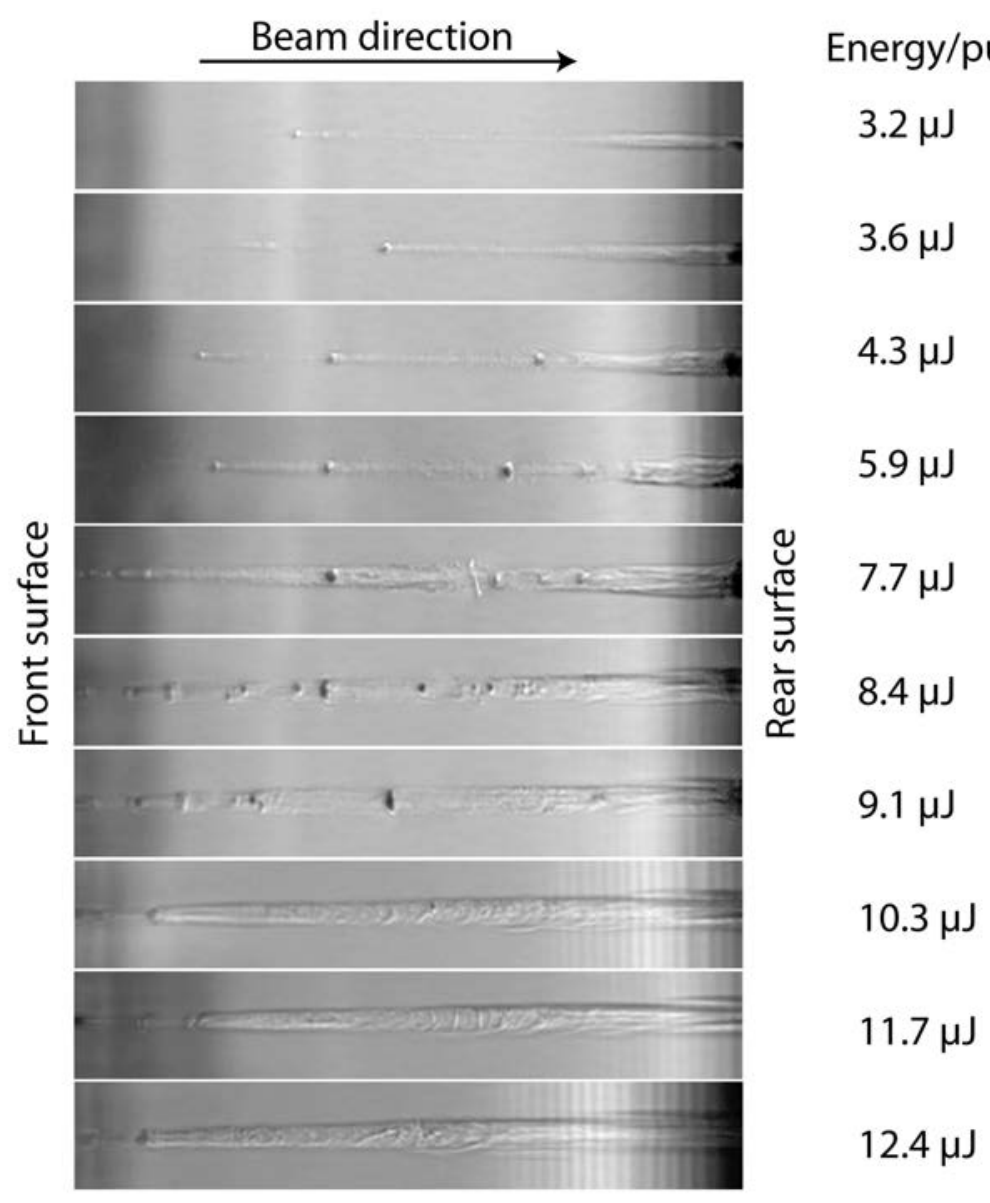

Figure 5 DIC images of the damage tracks produced by 1000 laser shots, at $100 \mathrm{~Hz}$, of $130 \mathrm{fs}$ pulse duration for different energies, at fixed Bessel beam onset $25 \mu \mathrm{m}$ before the sample entrance surface.

\section{CONCLUSION}

We have experimentally investigated the nonlinear propagation of Bessel filaments for high aspect ratio laser micromachining of glass. Microchannel drilling with aspect ratio up to 40 has been exhibited. Two different regimes have been identified. We investigated the dependence of the unstable filamentation regime on energy and beam onset and showed the disappearance of unstability at high energies.

*francois.courvoisier@femto-st.fr, phone +33 3816664 01, fax +33 381666423 


\section{REFERENCES}

[1] Whitesides, G. M. "The origins and the future of microfluidics" Nature 442, 368-373 (2006)

[2] Craighead, H. "Future lab-on-a-chip technologies for interrogating individual molecules," Nature 442, 387-393 (2006)

[3] Kovarik, M. L. and Jacobson, S. C. "Attoliter-scale dispensing in nanofluidic channels," Anal. Chem. 79, 16551660 (2007)

[4] Xia, Y. and Whitesides, G. M. "Soft lithography," Ann. Rev. of Mat. Sci. 28, 153-184 (1998)

[5] Psaltis, D., Quake, S. and Yang, C. "Developing optofluidic technology through the fusion of microfluidics and optics," Nature 442, 381-386 (2006)

[6] Della Valle, G., Osellame, R. and Laporta, P. "Micromachining of photonic devices by femtosecond laser pulses," J. Opt. A 11, 013001 (2009)

[7] Gattass, R. R. and Mazur, E. "Femtosecond laser micromachining in transparent materials," Nature Photon. 2, 219-225 (2008)

[8] Marcinkevičius, A., Juodkazis, S., Watanabe, M., Miwa, M., Matsuo, S., Misawa, H. and Nishii, J. "Femtosecond laser-assisted three-dimensional microfabrication in silica," Opt. Lett. 26, 277-279 (2001)

[9] Wortmann, D., Gottmann, J., Brandt, N. and Horn-Solle, H. "Micro- and nanostructures inside sapphire by fslaser irradiation and selective etching," Opt. Express 16, 1517-1522 (2008)

[10]Li, Y., Itoh, K., Watanabe, W., Yamada, K., Kuroda, D., Nishii, J. and Jiang, Y. "Three-dimensional hole drilling of silica glass from the rear surface with femtosecond laser pulses," Opt. Lett. 26, 1912-1914 (2001)

[11] Hwang, D., Choi, T. and Grigoropoulos, C. Liquid-assisted femtosecond laser drilling of straight and threedimensional microchannels in glass Appl. Phys. A 79, 605-612 (2004)

[12] Durnin, J., Miceli, J. and Eberly, J. H. "Diffraction free beams," Phys. Rev. Lett. 58, 1499-1501 (1987)

[13] Matsuoka, Y., Kizuka, Y. and Inoue, T. "The characteristics of laser micro drilling using a Bessel beam", Appl. Phys. A 84, 423-430 (2006)

[14] Amako, J., Sawaki, D. and Fujii, E. "Microstructuring transparent materials by use of nondiffracting ultrashort pulse beams generated by diffractive optics,'J. Opt. Soc. Am. B 20, 2562-2568 (2003)

[15] Papazoglou, D. G., Zergioti, I. and Tzortzakis, S. "Plasma strings from ultraviolet laser filaments drive permanent structural modifications in fused silica," Opt. Lett. 32, 2055-2057 (2007)

[16] Schwarz, J. and Diels, J. C. "UV filaments and their application for laser-induced lightning and high-aspectratio hole drilling" Appl. Phys. A 77, 185-191 (2003)

[17] Guyon, L., Courvoisier, F., Boutou, V., Nuter, R., Vincotte, A., Champeaux, S., Berge, L., Glorieux, P. and Wolf, J. P. "Self-organized bundle of lasing filaments in dense media," Phys. Rev. A 73, 051802 (2006)

[18] Couairon, A. and Mysyrowicz, A. "Femtosecond filamentation in transparent media," Phys. Rep 441, 47-189 (2007)

[19] Polesana, P., Faccio, D., Di Trapani, P., Dubietis, A., Piskarskas, A., Couairon, A. and Porras, M. A. "High localization, focal depth and contrast by means of nonlinear Bessel Beams," Opt. Express 13, 6160-6167 (2005)

[20] Polesana, P., Franco, M., Couairon, A., Faccio, D. and Di Trapani, P. "Filamentation in Kerr media from pulsed Bessel beams" Phys. Rev. A 77, 043814 (2008)

[21] Polynkin, P., Kolesik, M., Roberts, A., Faccio, D., Di Trapani, P. and Moloney, J. "Generation of extended plasma channels in air using femtosecond Bessel beams" Opt. Express 16, 15733-15740 (2008)

[22] Porras, M. A., Parola, A., Faccio, D., Dubietis, A. and Di Trapani, P. "Nonlinear unbalanced bessel beams: Stationary conical waves supported by nonlinear losses," Phys. Rev. Lett. 93, 153902 (2004)

[23] Bhuyan, M. K., Courvoisier, F., Lacourt, P.-A., Jacquot, M. ,Furfaro, L., Withford, M. J. and Dudley, J. M., "High aspect ratio taper-free microchannel fabrication using femtosecond Bessel beams," Opt. Express 18, 566$574(2010)$ 